Maltepe Journal of Mathematics

ISSN:2667-7660, URL:HTTP://DERGIPARK.ORG.TR/TR/PUB/MJM

Volume III Issue 1 (2021), Pages 6-14. Doi:https://DOI.org/10.47087/MJM.853437

\title{
REDUCTION METHOD FOR FUNCTIONAL NONCONVEX DIFFERENTIAL INCLUSIONS
}

\author{
HANANE CHOUIAL* AND MUSTAPHA FATEH YAROU** \\ *DEPARTMENT OF MATHEMATICS, LMPA LABORATORY, JIJEL UNIVERSITY, \\ ALGERIA, ORCID ID: 0000-0001-6599-9765 \\ **DEPARTMENT OF MATHEMATICS, LMPA LABORATORY, JIJEL UNIVERSITY, \\ ALGERIA, ORCID ID: 0000-0003-4083-1813
}

\begin{abstract}
Our aim in this paper is to present a reduction method that solves first order functional differential inclusion in the nonconvex case. This approach is based on a discretization of the time interval, a construction of approximate solutions by reducing the problem to a problem without delay and an application of known results in this case. We generalize earlier results, the right hand side of the inclusion has nonconvex values and satisfies a linear growth condition instead to be integrably bounded. The lack of convexity is replaced by the topological properties of decomposable sets, that represents a good alternative in the absence of convexity.
\end{abstract}

\section{INTRODUCTION}

Let $\tau, T \geq 0$, be two non-negative real numbers, $\mathcal{C}_{T}:=\mathcal{C}_{\mathbb{R}^{n}}([-\tau, T])$ is the Banach space of all continuous mappings from $[-\tau, 0]$ to $\mathbb{R}^{n}$ equipped with the norm of uniform convergence and $F:[0, T] \times \mathcal{C}_{0} \rightarrow \mathbb{R}^{n}$ be a set-valued mapping with nonempty closed values. In this work, we study the existence of solutions for the following differential inclusion with delay

$$
(\mathcal{D P}) \quad\left\{\begin{array}{lr}
\dot{x}(t) \in F(t, \mathcal{T}(t) x) & \text { a.e. } t \in[0, T] \\
x(t)=\varphi(t) & t \in[-\tau, 0]
\end{array}\right.
$$

where $\varphi \in \mathcal{C}_{0}$ and $\mathcal{T}(t): \mathcal{C}_{T} \longrightarrow \mathcal{C}_{0}$ defined by $\mathcal{T}(t) x(s)=x(t+s), \quad \forall s \in$ $[-\tau, 0]$. In [11], Fryszkowski proved an existence result for $(\mathcal{D P})$ when $F$ is a set-valued mapping with nonconvex values, measurable, integrably bounded and lower semicontinuous in $x$. The proof of this theorem is based on the construction of a continuous selection for a class of nonconvex set-valued mapping. The existence of such selection is proved in [10. In [12, Fryszkowski and Gorniewicz proved an

2020 Mathematics Subject Classification. Primary: 34K09 ; Secondaries: 49J52 .

Key words and phrases. Lower semicontinuous; nonconvex differential inclusion; reduction; delay; linear growth condition.

(C)2021 Maltepe Journal of Mathematics.

Submitted on January 4th, 2021. Published on April 30th, 2021. Communicated by Valéria Neves DOMINGOS CAVALCANTİ .. 
existence result for differential inclusion of the form

$$
(\mathcal{P}) \quad\left\{\begin{array}{l}
\dot{x}(t) \in F(t, x(t)) \quad \text { a.e. } t \in[0, T] \\
x(0)=x_{0},
\end{array}\right.
$$

where $F$ is a set-valued mapping measurable in $(t, x)$ and lower semicontinuous in $x$ with nonconvex values, satisfying a linear growth condition. The main tool used in their proof is a continuous selection theorem for the set-valued mapping

$$
K_{F}(x)=\left\{y \in L_{\mathbb{R}^{n}}^{1}([0, T]): y(t) \in F(t, x(t)) \quad \text { a.e. on }[0, T]\right\},
$$

which is well defined on $\mathcal{C}_{\mathbb{R}^{n}}([0, T])$ and is lower semicontinuous with decomposable values. Decomposable sets represent a good alternative in the absence of convexity. Our aim in this work, is to prove a general existence result for $(\mathcal{D P})$, where $F$ satisfies a linear growth condition instead to be integrably bounded, that is

$$
\|y\| \leq(1+\|\varphi\|) \rho(t) \text {, for every } y \in F(t, \varphi) \text { and }(t, \varphi) \in[0, T] \times \mathcal{C}_{0} .
$$

We extend also the existence result for the Cauchy problem without delay in the nonconvex case. Some applications have been obtained by considering such delayed set-valued mapping as perturbations (external forces applied) on systems governed by subdifferential operators, particularly in the case of the so-called Sweeping process, see for instance [7], 8]. We refer to [1]-3] for recent results, 44, [5] and [13] for other approaches. The paper is organized as follows. In Section 2, we recall concepts and preliminaries needed in the paper. In Section 3, we provide the existence result for problem $(\mathcal{D P})$.

\section{Preliminaries}

Throughout the paper, we will use the following notations and definitions. Let $\mathbb{R}^{n}$ be the $n$ dimensional Euclidean space and $\|\cdot\|$ its norm. $\mathcal{C}_{T}:=\mathcal{C}_{\mathbb{R}^{n}}([-\tau, T])$ is the Banach space of all continuous mappings from $[-\tau, T]$ to $\mathbb{R}^{n}$ endowed with the sup-norm, $L_{\mathbb{R}^{n}}^{1}([0, T])$ is the Banach space of all measurable mappings from $[0, T]$ to $\mathbb{R}^{n}$. Let $\mathcal{B}\left(\mathcal{C}_{0}\right)$ be the $\sigma$-algebra of Borel sets of $\mathcal{C}_{0}$ and $\mathcal{L}$ the $\sigma$-algebra of Lebesgue measurable subsets of $[0, T], d(x, A)$ mean the usual distance from a point $x$ to a set $A$, i.e., $d(x, A):=\inf _{y \in A}\|x-y\|$. A set-valued mapping $F:[0, T] \times \mathcal{C}_{0} \rightarrow \mathbb{R}^{n}$ is integrably bounded if there exists an integrable function $\rho:[0, T] \rightarrow \mathbb{R}^{+}$such that

$$
\|F(t, \varphi)\|:=\sup \{\|y\| ; y \in F(t, \varphi)\} \leq \rho(t), t \in[0, T], \varphi \in \mathcal{C}_{0} .
$$

Definition 2.1. ([6]) Let $X$ and $Y$ be two topological spaces, $F: X \rightarrow Y$ a setvalued mapping with closes valued, is called lower semicontinuous (lsc for short) at a point $x_{0} \in X$ if for any $y_{0} \in F\left(x_{0}\right)$ and any neighborhood $U$ of $y_{0}$ such that $F\left(x_{0}\right) \cap U \neq \emptyset$, there exists a neighborhood $V\left(x_{0}\right)$ of the point $x_{0}$ such that $F\left(x_{0}\right) \cap U \neq \emptyset$ for all $x \in V\left(x_{0}\right)$. A set-valued mapping $F$ is said to be lower semicontinuous if it is so at every point $x_{0} \in X$.

If $X$ and $Y$ are metric spaces, it's equivalent to say: for each $x_{0} \in[0, T]$ and $y_{0} \in F\left(x_{0}\right)$ and any sequence $x_{n} \longrightarrow x_{0}$ there is $y_{n} \in F\left(x_{n}\right)$ such that $y_{n} \longrightarrow y_{0}$.

Lemma 2.1. (Gronwall inequality) Let $u, v:\left[t_{0}, t_{1}\right] \longrightarrow \mathbb{R}^{+}$two continuous functions such that, for any $C \geq 0$, we have

$$
u(t) \leq C+\int_{t_{0}}^{t} u(s) v(s) d s, \quad \forall t \in\left[t_{0}, t_{1}\right] .
$$


Then

$$
u(t) \leq C \exp \left(\int_{t_{0}}^{t} v(s) d s\right), \quad \forall t \in\left[t_{0}, t_{1}\right]
$$

\section{EXISTENCE OF SOLUTiOnS}

In this section, we begin by the following result for the undelayed problem due to Fryszkowski and Gorniewicz (see [12]).

Theorem 3.1. Let $G:[0, T] \times \mathbb{R}^{n} \rightarrow \mathbb{R}^{n}$ be a set-valued mapping with nonempty closed values satisfying

(i) $G$ is $\mathcal{L} \otimes \mathcal{B}\left(\mathbb{R}^{n}\right)$ measurable;

(ii) for every $t \in[0, T], G(t, \cdot)$ is lsc in $\mathbb{R}^{n}$;

(iii) there exists an integrable function $\rho:[0, T] \longrightarrow \mathbb{R}^{+}$such that

$$
\|y\| \leq(1+|x|) \rho(t), \text { for every } y \in G(t, x) \text { and }(t, x) \in[0, T] \times \mathbb{R}^{n} .
$$

Then, $\forall x_{0} \in \mathbb{R}^{n}$, the problem

$$
\left\{\begin{array}{l}
\dot{x}(t) \in G(t, x(t)) \quad \text { a.e. on }[0, T] \\
x(0)=x_{0}
\end{array}\right.
$$

admits at least one solution $x:[0, T] \rightarrow \mathbb{R}^{n}$ absolutely continuous on $[0, T]$.

The proof of this theorem is based on a selection theorem for decomposable sets stated in [11].

Now, we are able to give the existence result for the delayed problem.

Theorem 3.2. Let $F:[0, T] \times \mathcal{C}_{0} \rightarrow \mathbb{R}^{n}$ be a set-valued mapping with nonempty closed values such that

(i) $F$ is $\mathcal{L} \otimes \mathcal{B}\left(\mathcal{C}_{0}\right)$ measurable;

(ii) for every $t \in[0, T], F(t, \cdot)$ is lsc in $\mathcal{C}_{0}$;

(iii) for every $(t, \varphi) \in[0, T] \times \mathcal{C}_{0}$

$$
\|F(t, \varphi)\| \leq(1+\|\varphi(0)\|) \rho(t) .
$$

Then, $\forall \varphi \in \mathcal{C}_{0}$, the problem $(\mathcal{D P})$ admits at least one continuous solution $x$ : $[-\tau, T] \rightarrow \mathbb{R}^{n}$, absolutely continuous on $[0, T]$.

Proof. We will reduce our problem to a problem without delay and apply Theorem 3.1. For simplcity, we take $T=1$ and consider for every $n \in \mathbb{N}$ a partition of $[0, T]$ defined by $t_{i}^{n}=i \mu_{n} T, \mu_{n}=2^{-n}, i=0,1, \ldots \ldots, 2^{n}$.

Step 1 Construction of approximate solutions.

For every $(t, x) \in\left[-\tau, t_{1}^{n}\right] \times \mathbb{R}^{n}$, we define $f_{0}^{n}:\left[-\tau, t_{1}^{n}\right] \times \mathbb{R}^{n}$ by

$$
f_{0}^{n}(t, x)= \begin{cases}\varphi(t) & \text { if } t \in[-\tau, 0] ; \\ \varphi(0)+\frac{t}{\mu_{n}}(x-\varphi(0)) & \text { if } \left.t \in] 0, t_{1}^{n}\right]\end{cases}
$$

clearly $f_{0}^{n}\left(t_{1}^{n}, x\right)=x, \quad \forall x \in \mathbb{R}^{n}$.

We define the set-valued mapping $G_{0}^{n}$ on $\left[0, t_{1}^{n}\right] \times \mathbb{R}^{n}$ with closed values in $\mathbb{R}^{n}$ by

$$
G_{0}^{n}(t, x):=F\left(t, \mathcal{T}\left(t_{1}^{n}\right) f_{0}^{n}(\cdot, x)\right) \quad \forall(t, x) \in\left[0, t_{1}^{n}\right] \times \mathbb{R}^{n} .
$$


Let show that $G_{0}^{n}$ satisfies the conditions of Theorem 3.1. Remark first, that the function $x \longmapsto \mathcal{T}\left(t_{1}^{n}\right) f_{0}^{n}(\cdot, x)$ is Lipschitz. Indeed, for every $x, y \in \mathbb{R}^{n}$ we have

$$
\begin{aligned}
\left\|\mathcal{T}\left(t_{1}^{n}\right) f_{0}^{n}(\cdot, x)-\mathcal{T}\left(t_{1}^{n}\right) f_{0}^{n}(\cdot, y)\right\|_{\mathcal{C}_{0}} & =\sup _{s \in[-\tau, 0]}\left\|f_{0}^{n}\left(t_{1}^{n}+s, x\right)-f_{0}^{n}\left(t_{1}^{n}+s, y\right)\right\| \\
& =\sup _{s \in\left[-\mu_{n}, 0\right]}\left\|f_{0}^{n}\left(t_{1}^{n}+s, x\right)-f_{0}^{n}\left(t_{1}^{n}+s, y\right)\right\| \\
& =\sup _{s \in\left[-\mu_{n}, 0\right]}\left\|\frac{t_{1}^{n}+s}{\mu_{n}}(x-y)\right\| \\
& =\|x-y\| .
\end{aligned}
$$

The measurability and lower semicontinuity of $G$ follows from that of $F$. Furthermore, by the condition iii) of Theorem 3.2 we have, for every $t \in\left[0, t_{1}^{n}\right]$ and $x \in \mathbb{R}^{n}$,

$$
\begin{aligned}
\left\|G_{0}^{n}(t, x)\right\|=\left\|F\left(t, \mathcal{T}\left(t_{1}^{n}\right) f_{0}^{n}(\cdot, x)\right)\right\| & \leq\left(1+\left\|\mathcal{T}\left(t_{1}^{n}\right) f_{0}^{n}(0, x)\right\|\right) \rho(t) \\
& =\left(1+\left\|f_{0}^{n}\left(t_{1}^{n}, x\right)\right\|\right) \rho(t) \\
& =(1+\|x\|) \rho(t) .
\end{aligned}
$$

Hence $G_{0}^{n}$ verifies the conditions of Theorem 3.1, this provides an absolutely continuous solution $v_{0}^{n}:\left[0, t_{1}^{n}\right] \longrightarrow \mathbb{R}^{n}$ to the problem

$$
\left\{\begin{aligned}
\dot{v}_{0}^{n}(t) & \in G_{0}^{n}\left(t, v_{1}^{n}(t)\right) & & \text { a.e. on }\left[0, t_{1}^{n}\right] ; \\
v_{0}^{n}(t) & =\varphi(0)+\int_{0}^{t} \dot{v}_{0}^{n}(s) d s & & \left.\forall t \in] 0, t_{1}^{n}\right] ; \\
v_{0}^{n}(0) & =\varphi(0) . & &
\end{aligned}\right.
$$

That is, $v_{0}^{n}$ is a solution to

$$
\begin{cases}\dot{v}_{0}^{n}(t) & \in F\left(t, \mathcal{T}\left(t_{1}^{n}\right) f_{0}^{n}(\cdot, x)\right) \quad \text { a. e. on }\left[0, t_{1}^{n}\right] \\ v_{0}^{n}(0) & =\varphi(0) .\end{cases}
$$

Put

$$
x_{n}(t)= \begin{cases}\varphi(t) & \text { if } t \in[-\tau, 0] \\ v_{0}^{n}(t) & \text { if } \left.t \in] 0, t_{1}^{n}\right]\end{cases}
$$

As before, for every $(t, x) \in\left[-\tau, t_{1}^{n}\right] \times \mathbb{R}^{n}$, we define $f_{1}^{n}:\left[-\tau, t_{2}^{n}\right] \times \mathbb{R}^{n} \longrightarrow \mathbb{R}^{n}$ by

$$
f_{1}^{n}(t, x)= \begin{cases}x_{n}(t) & \text { if } t \in\left[-\tau, t_{1}^{n}\right] \\ x_{n}\left(t_{1}^{n}\right)+\frac{t-t_{1}^{n}}{\mu_{n}}\left(x-x_{n}\left(t_{1}^{n}\right)\right) & \text { if } \left.t \in] t_{1}^{n}, t_{2}^{n}\right] .\end{cases}
$$

with $f_{1}^{n}\left(t_{2}^{n}, x\right)=x, \forall x \in \mathbb{R}^{n}$. Hence, we can define similarly the set-valued mapping $G_{1}^{n}$ on $\left[t_{1}^{n}, t_{2}^{n}\right] \times \mathbb{R}^{n}$ with closed values of $\mathbb{R}^{n}$ by

$$
G_{1}^{n}(t, x):=F\left(t, \mathcal{T}\left(t_{1}^{n}\right) f_{1}^{n}(\cdot, x)\right) \quad \forall(t, x) \in\left[t_{1}^{n}, t_{2}^{n}\right] \times \mathbb{R}^{n}
$$

satisfying for every $t \in\left[t_{1}^{n}, t_{2}^{n}\right]$ and $x \in \mathbb{R}^{n}$,

$$
\begin{aligned}
\left\|G_{1}^{n}(t, x)\right\|=\left\|F\left(t, \mathcal{T}\left(t_{2}^{n}\right) f_{1}^{n}(\cdot, x)\right)\right\| & \leq\left(1+\left\|\mathcal{T}\left(t_{2}^{n}\right) f_{1}^{n}(0, x)\right\|\right) \rho(t) \\
& =\left(1+\left\|f_{1}^{n}\left(t_{2}^{n}, x\right)\right\|\right) \rho(t) \\
& =(1+\|x\|) \rho(t) .
\end{aligned}
$$


The function $x \longmapsto \mathcal{T}\left(t_{2}^{n}\right) f_{1}^{n}(\cdot, x)$ is Lipschitz since for all $x, y \in \mathbb{R}^{n}$ we have

$$
\begin{aligned}
\left\|\mathcal{T}\left(t_{2}^{n}\right) f_{1}^{n}(\cdot, x)-\mathcal{T}\left(t_{2}^{n}\right) f_{1}^{n}(\cdot, y)\right\| & =\sup _{s \in[-\tau, 0]}\left\|f_{1}^{n}\left(t_{2}^{n}+s, x\right)-f_{1}^{n}\left(t_{2}^{n}+s, y\right)\right\| \\
& =\sup _{s \in\left[-\mu_{n}, 0\right]}\left\|f_{1}^{n}\left(t_{2}^{n}+s, x\right)-f_{1}^{n}\left(t_{2}^{n}+s, y\right)\right\| \\
& =\sup _{s \in\left[-\mu_{n}, 0\right]} \| x_{n}\left(t_{1}^{n}\right)+\frac{t_{2}^{n}+s-t_{1}^{n}}{\mu_{n}}\left(x-x_{n}\left(t_{1}^{n}\right)\right) \\
& -\left(x_{n}\left(t_{1}^{n}\right)+\frac{t_{2}^{n}+s-t_{1}^{n}}{\mu_{n}}\left(y-x_{n}\left(t_{1}^{n}\right)\right)\right) \| \\
& =\sup _{s \in\left[-\mu_{n}, 0\right]}\left\|\frac{t_{2}^{n}+s-t_{1}^{n}}{2-n}(x-y)\right\| \\
& =\left\|\frac{t_{2}^{n}-t_{1}^{n}}{\mu_{n}}(x-y)\right\| \\
& =\|x-y\| .
\end{aligned}
$$

Hence $G_{1}^{n}$ verifies the conditions of Theorem 3.1, this provides an absolutely continuous solution $v_{1}^{n}:\left[t_{1}^{n}, t_{2}^{n}\right] \longrightarrow \mathbb{R}^{n}$ to the problem

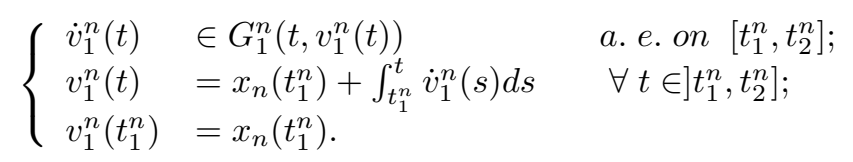

So $v_{1}^{n}$ is a solution of

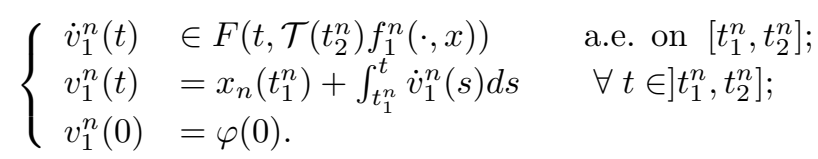

By induction, suppose that $x_{n}$ is defined on $\left[-\tau, t_{k}^{n}\right]$, absolutely continuous on $\left[0, t_{k}^{n}\right]$, and satisfies

$$
\left\{\begin{array}{lll}
\dot{x}_{n}(t) & \in F\left(t, \mathcal{T}\left(t_{k-1}^{n}\right) f_{k-1}^{n}(\cdot, x)\right) & \text { a.e. on }\left[t_{k-1}^{n}, t_{k}^{n}\right] ; \\
x_{n}(t) & =x_{n}\left(t_{k-1}^{n}\right)+\int_{t_{k-1}^{n}}^{t} \dot{x}_{n}(s) d s & \left.\forall t \in] t_{k-1}^{n}, t_{k}^{n}\right]
\end{array}\right.
$$

and build a solution on $\left[t_{k}^{n}, t_{k+1}^{n}\right]$. For every $(t, x) \in\left[-\tau, t_{1}^{n}\right] \times \mathbb{R}^{n}$, we defined $f_{k}^{n}:\left[-\tau, t_{k+1}^{n}\right] \times \mathbb{R}^{n} \longrightarrow \mathbb{R}^{n}$ by

$$
f_{k}^{n}(t, x)= \begin{cases}x_{n}(t) & \text { if } t \in\left[-\tau, t_{k}^{n}\right] \\ x_{n}\left(t_{k}^{n}\right)+\frac{t-t_{k}^{n}}{\mu_{n}}\left(x-x_{n}\left(t_{k}^{n}\right)\right) & \text { if } \left.t \in] t_{k}^{n}, t_{k+1}^{n}\right]\end{cases}
$$

with $f_{k}^{n}\left(t_{k+1}^{n}, x\right)=x$ and $f_{k}^{n} \in \mathcal{C}_{\mathbb{R}^{n}}\left(\left[-\tau, t_{k+1}^{n}\right]\right)$. The function $x \longmapsto \mathcal{T}\left(t_{k+1}^{n}\right) f_{k}^{n}(\cdot, x)$ is Lipschitz. Indeed, for all $x, y \in \mathbb{R}^{n}$ we have

$$
\begin{gathered}
\left\|\mathcal{T}\left(t_{k+1}^{n}\right) f_{k}^{n}(\cdot, x)-\mathcal{T}\left(t_{k+1}^{n}\right) f_{k}^{n}(\cdot, y)\right\|= \\
\sup _{s \in[-\tau, 0]}\left\|f_{k}^{n}\left(t_{k+1}^{n}+s, x\right)-f_{k}^{n}\left(t_{k+1}^{n}+s, y\right)\right\| \\
=\sup _{t \in\left[-\tau+t_{k+1}^{n}, t_{k+1}^{n}\right]}\left\|f_{k}^{n}(t, x)-f_{k}^{n}(t, y)\right\| .
\end{gathered}
$$

We distinguish two cases

(1) if $-\tau+t_{k+1}^{n} \leq t_{k}^{n}$, we have

$$
\begin{aligned}
\sup _{t \in\left[-\tau+t_{k+1}^{n}, t_{k+1}^{n}\right]}\left\|f_{k}^{n}(t, x)-f_{k}^{n}(t, y)\right\| & =\sup _{t \in\left[t_{k}^{n}, t_{k+1}^{n}\right]}\left\|f_{k}^{n}(t, x)-f_{k}^{n}(t, y)\right\| \\
& =\sup _{t \in\left[t_{k}^{n}, t_{k+1}^{n}\right]}\left\|\frac{t-t_{k}^{n}}{\mu_{n}}(x-y)\right\| \\
& =\|x-y\| .
\end{aligned}
$$


(2) if $t_{k}^{n} \leq-\tau+t_{k+1}^{n} \leq t_{k+1}^{n}$, we have

$$
\begin{aligned}
\sup _{t \in\left[-\tau+t_{k+1}^{n}, t_{k+1}^{n}\right]}\left\|f_{k}^{n}(t, x)-f_{k}^{n}(t, y)\right\| & \leq \sup _{t \in\left[t_{k}^{n}, t_{k+1}^{n}\right]}\left\|f_{k}^{n}(t, x)-f_{k}^{n}(t, y)\right\| \\
& =\sup _{t \in\left[t_{k}^{n}, t_{k+1}^{n}\right]}\left\|\frac{t-t_{k}^{n}}{\mu_{n}}(x-y)\right\| \\
& =\|x-y\| .
\end{aligned}
$$

Similarly we can define $G_{k}^{n}$ on $\left[t_{k}^{n}, t_{k+1}^{n}\right] \times \mathbb{R}^{n}$ with closed values of $\mathbb{R}^{n}$ by

$$
G_{k}^{n}(t, x):=F\left(t, \mathcal{T}\left(t_{k+1}^{n}\right) f_{k}^{n}(\cdot, x)\right) \quad \forall(t, x) \in\left[t_{k}^{n}, t_{k+1}^{n}\right] \times \mathbb{R}^{n}
$$

satisfying conditions of Theorem 3.1. Hence, there exists an absolutely continuous solution $v_{k}^{n}:\left[t_{k}, t_{k+1}\right] \longrightarrow \mathbb{R}^{n}$ to

$$
\left\{\begin{array}{llr}
\dot{v}_{k}^{n}(t) & \in G_{k}^{n}\left(t, v_{k}^{n}(t)\right) & \text { a.e. on }\left[t_{k}^{n}, t_{k+1}^{n}\right] ; \\
v_{k}^{n}(t) & =x_{n}\left(t_{k}^{n}\right)+\int_{t_{k}^{n}}^{t} \dot{v}_{k}^{n}(s) d s & \forall t \in\left[t_{k}^{n}, t_{k+1}^{n}\right] ; \\
v_{k}^{n}\left(t_{k}^{n}\right) & =x_{n}\left(t_{k}^{n}\right) . &
\end{array}\right.
$$

So $v_{k}^{n}$ is a solution of

$$
\left\{\begin{array}{llr}
\dot{v}_{k}^{n}(t) & \in F\left(t, \mathcal{T}\left(t_{k+1}^{n}\right) f_{k}^{n}(\cdot, x)\right) & \text { a.e. on }\left[t_{k}^{n}, t_{k+1}^{n}\right] ; \\
v_{k}^{n}(t) & =x_{n}\left(t_{k}^{n}\right)+\int_{t_{k}^{n}}^{t} \dot{v}_{k}^{n}(s) d s & \forall t \in\left[t_{k}^{n}, t_{k+1}^{n}\right] ; \\
v_{k}^{n}\left(t_{k}^{n}\right) & =x_{n}\left(t_{k}^{n}\right) . &
\end{array}\right.
$$

Putting $x_{n}(t)=v_{k}^{n}(t)$ on $\left[t_{k}^{n}, t_{k+1}^{n}\right]$, we obtain

$$
x_{n}(t)=\left\{\begin{array}{cl}
v_{0}^{n}(t)=\varphi(0)+\int_{0}^{t} \dot{x}_{n}(s) d s & \text { if } t \in\left[0, t_{1}^{n}\right] ; \\
v_{1}^{n}(t)=x_{n}\left(t_{1}^{n}\right)+\int_{t_{1}^{n}}^{t} \dot{x}_{n}(s) d s & \text { if } t \in\left[t_{1}^{n}, t_{2}^{n}\right] ; \\
\cdots & \\
v_{k}^{n}(t)=x_{n}\left(t_{k}^{n}\right)+\int_{t_{k}^{n}}^{t} \dot{x}_{n}(s) d s & \text { if } t \in\left[t_{k}^{n}, t_{k+1}^{n}\right] .
\end{array}\right.
$$

For every $t \in[0,1]$, we set $\left.\left.\theta_{n}(t)=t_{i}^{n}, \delta_{n}(t)=t_{i+1}^{n}, \forall t \in\right] t_{i}^{n}, t_{i+1}^{n}\right]$ and $\theta_{n}(0)=0$ and define $f_{\mu_{n} \delta_{n}(t)-1}^{n} \in \mathcal{C}_{\mathbb{R}^{n}\left(\left[-\tau, \delta_{n}(t)\right]\right)}$ by

$$
f_{\mu_{n} \delta_{n}(t)-1}^{n}(t, x)= \begin{cases}x_{n}(t) & \text { if } t \in\left[-\tau, \theta_{n}(t)\right] ; \\ x_{n}\left(\theta_{n}(t)\right)+\frac{t-\theta_{n}(t)}{\mu_{n}}\left(x-x_{n}\left(\theta_{n}(t)\right)\right) & \text { if } \left.t \in] \theta_{n}(t), \delta_{n}(t)\right] .\end{cases}
$$

Clearly $x_{n}$ is continuous on $[-\tau, 1]$, absolutely continuous on $[0,1]$ and satisfies

$$
\left\{\begin{array}{llrl}
\dot{x}_{n}(t) & \in F\left(t, \mathcal{T}\left(\delta_{n}(t)\right) f_{\mu_{n} \delta_{n}(t)-1}^{n}\left(\cdot, x_{n}(t)\right)\right) & \text { a. e. on }[0,1] ; \\
x_{n}(t)=\varphi(0)+\int_{0}^{t} \dot{x}_{n}(s) d s & \forall t \in[0,1] ; \\
x_{n}(t)=\varphi(t) & \forall t \in[-\tau, 0] .
\end{array}\right.
$$

Step 2 Uniform convergence.

By the condition iii) of Theorem 3.1 and $(3.2$, for almost $t \in[0,1]$, one has

$$
\dot{x}_{n}(t) \in F\left(t, \mathcal{T}\left(\delta_{n}(t)\right) f_{\mu_{n} \delta_{n}(t)-1}^{n}\left(\cdot, x_{n}(t)\right)\right),
$$

with $\mathcal{T}\left(\delta_{n}(t)\right) f_{\mu_{n} \delta_{n}(t)-1}^{n}\left(\cdot, x_{n}(t)\right)(0)=x_{n}(t)$ and

$$
\left\|F\left(t, \mathcal{T}\left(\delta_{n}(t)\right) f_{\mu_{n} \delta_{n}(t)-1}^{n}\left(\cdot, x_{n}(t)\right)\right)\right\| \leq\left(1+\left\|x_{n}(t)\right\|\right) \rho(t) .
$$


Further, since $x_{n}$ is absolutely continuous on $[0,1]$, we have

$$
\begin{aligned}
\left\|x_{n}(t)-\varphi(0)\right\| & \leq \int_{0}^{t}\left\|\dot{x}_{n}(s)\right\| d s \\
& \leq \int_{0}^{t}\left(1+\left\|x_{n}(s)\right\|\right) \rho(s) d s \\
& \leq \int_{0}^{t}\left(1+\left\|x_{n}(s)\right\| \rho(s)\right) d s \\
& =\int_{0}^{t} \rho(s) d s+\int_{0}^{t} \rho(s)\left\|x_{n}(s)\right\| d s, \quad \forall t \in[0,1] .
\end{aligned}
$$

Then, $\left\|x_{n}(t)\right\| \leq\|\varphi(0)\|+\int_{0}^{t} \rho(s) d s+\int_{0}^{t} \rho(s)\left\|x_{n}(s)\right\| d s, \forall t \in[0,1]$. Using Lemma 2.1. we obtain for all $t \in[0,1]$,

$$
\left\|x_{n}(t)\right\| \leq\left(\|\varphi(0)\|+\int_{0}^{t} \rho(s) d s\right) \exp \left(\int_{0}^{t} \rho(s) d s\right) .
$$

Let $\alpha(t)=\left(\|\varphi(0)\|+\int_{0}^{t} \rho(s) d s\right) \exp \left(\int_{0}^{t} \rho(s) d s\right)$. Hence for almost every $t \in[0,1]$,

$$
\left\|\dot{x}_{n}(t)\right\| \leq(1+\alpha(t)) \rho(t)
$$

By $(3.3),\left(\dot{x}_{n}(t)\right)_{n}$ is relatively compact in $L_{\mathbb{R}^{n}}^{1}([0,1])$. By extracting a subsequence, we may assume that $\left(\dot{x}_{n}\right)_{n}$ converges $\sigma\left(L^{1}, L^{\infty}\right)$ to some $y \in L_{\mathbb{R}^{n}}^{1}([0,1])$. On the other hand, by (3.3) again, $\left(x_{n}\right)_{n}$ is equi-continuous, Ascoli's Theorem yields that $\left(x_{n}\right)_{n}$ converges uniformly in $[0,1]$ to $x$ and

$$
x(t)=\varphi(0)+\int_{0}^{t} y(s) d s, \forall t \in[0,1]
$$

hence $\dot{x}(t)=y(t)$ almost everywhere. Now, let show that

$$
\begin{gathered}
\left\|\mathcal{T}\left(\delta_{n}(t)\right) f_{\mu_{n} \delta_{n}(t)-1}^{n}\left(\cdot, x_{n}(t)\right)-\mathcal{T}(t) x\right\| \longrightarrow 0, \text { when } n \longrightarrow \infty . \\
\sup _{s \in[-\tau, 0]}\left\|\mathcal{T}\left(\delta_{n}(t)\right) f_{\mu_{n} \delta_{n}(t)-1}^{n}\left(s, x_{n}(t)\right)-\mathcal{T}(t) x(s)\right\|_{\mathcal{C}_{0}}= \\
\sup _{s \in[-\tau, 0]}\left\|f_{\mu_{n} \delta_{n}(t)-1}^{n}\left(\delta_{n}(t)+s, x_{n}(t)\right)-x(s+t)\right\| \\
=\sup _{s \in[-\tau, 0]}\left\|f_{\mu_{n} \delta_{n}(t)-1}^{n}\left(\delta_{n}(t)+s, x_{n}(t)\right)-x\left(\delta_{n}(t)+s\right)+x\left(\delta_{n}(t)+s\right)-x(s+t)\right\| \\
\leq \sup _{s \in[-\tau, 0]}\left\|f_{\mu_{n} \delta_{n}(t)-1}^{n}\left(\delta_{n}(t)+s, x_{n}(t)\right)-x\left(\delta_{n}(t)+s\right)\right\|+ \\
\sup _{s \in[-\tau, 0]}\left\|x\left(\delta_{n}(t)+s\right)-x(s+t)\right\| .
\end{gathered}
$$

firstly,

$$
\begin{gathered}
\sup _{s \in[-\tau, 0]}\left\|f_{\mu_{n} \delta_{n}(t)-1}^{n}\left(\delta_{n}(t)+s, x_{n}(t)\right)-x\left(\delta_{n}(t)+s\right)\right\| \\
\leq \sup _{s \in\left[-\tau,-\mu_{n}\right]}\left\|f_{\mu_{n} \delta_{n}(t)-1}^{n}\left(\delta_{n}(t)+s, x_{n}(t)\right)-x\left(\delta_{n}(t)+s\right)\right\| \\
+\sup _{s \in\left[-\mu_{n}, 0\right]}\left\|f_{\mu_{n} \delta_{n}(t)-1}^{n}\left(\delta_{n}(t)+s, x_{n}(t)\right)-x\left(\delta_{n}(t)+s\right)\right\| \\
\quad=\sup _{s \in\left[-\tau,-\mu_{n}\right]}\left\|x_{n}\left(\delta_{n}(t)+s\right)-x\left(\delta_{n}(t)+s\right)\right\|+ \\
\sup _{s \in\left[-\mu_{n}, 0\right]}\left\|x_{n}\left(\theta_{n}(t)\right)+\frac{\delta_{n}(t)+s-\theta_{n}(t)}{\mu_{n}}\left(x_{n}(t)-x_{n}\left(\theta_{n}(t)\right)-x\left(\delta_{n}(t)+s\right)\right)\right\| \\
\quad=\sup _{s \in\left[-\tau,-\mu_{n}\right]}\left\|x_{n}\left(\delta_{n}(t)+s\right)-x\left(\delta_{n}(t)+s\right)\right\| \\
+\sup _{s \in\left[-\mu_{n}, 0\right]}\left\|\frac{s}{\mu_{n}}\left(x_{n}(t)-x_{n}\left(\theta_{n}(t)\right)\right)+x_{n}(t)-x\left(\delta_{n}(t)+s\right)\right\| \\
=\left\|x_{n}\left(\theta_{n}(t)\right)-x\left(\theta_{n}(t)\right)\right\|+\left\|x_{n}(t)-x_{n}\left(\delta_{n}(t)\right)\right\| .
\end{gathered}
$$


On the other hand

$$
\begin{aligned}
\sup _{s \in[-\tau, 0]}\left\|x\left(\delta_{n}(t)+s\right)-x(s+t)\right\| \leq & \sup _{s \in\left[-\tau,-\mu_{n}\right]}\left\|x\left(\delta_{n}(t)+s\right)-x(s+t)\right\| \\
& +\sup _{s \in\left[-\mu_{n}, 0\right]}\left\|x\left(\delta_{n}(t)+s\right)-x(s+t)\right\| \\
= & \sup _{\substack{s \in\left[-\tau,-\mu_{n}\right]\\
}}\left\|x\left(\delta_{n}(t)+s\right)-x(s+t)\right\| \\
& +\left\|x\left(\delta_{n}(t)\right)-x(t)\right\| .
\end{aligned}
$$

Then

$$
\begin{aligned}
& \sup _{s \in[-\tau, 0]}\left\|\mathcal{T}\left(\delta_{n}(t)\right) f_{\mu_{n} \delta_{n}(t)-1}^{n}\left(s, x_{n}(t)\right)-\mathcal{T}(t) x(s)\right\|_{\mathcal{C}_{0}} \leq \\
& \quad\left\|x_{n}\left(\theta_{n}(t)\right)-x\left(\theta_{n}(t)\right)\right\|+\left\|x_{n}(t)-x_{n}\left(\delta_{n}(t)\right)\right\|+ \\
& \sup _{s \in\left[-\tau,-\mu_{n}\right]}\left\|x\left(\delta_{n}(t)+s\right)-x(s+t)\right\|+\left\|x\left(\delta_{n}(t)\right)-x(t)\right\| .
\end{aligned}
$$

As $\left|\theta_{n}(t)-t\right| \leq \mu_{n}$ and $\left|\delta_{n}(t)-t\right| \leq \mu_{n}, \forall t \in[0,1]$ then $\theta_{n}(t) \longrightarrow t$ and $\delta_{n}(t) \longrightarrow t$ for $n$ large enough. Furthermore, $\left(x_{n}\right)_{n}$ converges uniformly to $x, \| x\left(\delta_{n}(t)\right)-$ $x(t)\|\longrightarrow 0,\| x_{n}\left(\delta_{n}(t)\right)-x_{n}(t) \| \longrightarrow 0$ and $\left\|x_{n}\left(\theta_{n}(t)\right)-x\left(\theta_{n}(t)\right)\right\| \longrightarrow 0$. As $x$ is uniformly continuous, there is $\lambda>0$ such that $|s-t| \leq \lambda$ implies $\|x(s)-x(t)\| \leq \epsilon$. But we have $\left|\delta_{n}(t)+s-(s+t)\right| \leq \mu_{n}$ for all $s \in\left[-\tau, \mu_{n}\right]$. Hence

$$
\sup _{s \in\left[-\tau,-\mu_{n}\right]}\left\|x\left(\delta_{n}(t)+s\right)-x(s+t)\right\| \leq \epsilon \text { for } \lambda \leq \mu_{n} .
$$

We can conclude that $\mathcal{T}\left(\delta_{n}(t)\right) f_{\mu_{n} \delta_{n}(t)-1}^{n}\left(\cdot, x_{n}(t)\right) \longrightarrow \mathcal{T}(t) x$ in $\mathcal{C}_{0}$.

Finally, since $\mathcal{T}\left(\delta_{n}(t)\right) f_{\mu_{n} \delta_{n}(t)-1}^{n}\left(\cdot, x_{n}(t)\right) \longrightarrow \mathcal{T}(t) x$ in $\mathcal{C}_{0},\left(\dot{x}_{n}\right)_{n}$ converges $\sigma\left(L^{1}, L^{\infty}\right)$ to $\dot{x} \in L_{\mathbb{R}^{n}}^{1}([0,1])$ and the set-valued mapping $F(t, \cdot)$ is lsc with closed values on $\mathcal{C}_{0}$, then $\dot{x}(t) \in F(t, \mathcal{T}(t) x)$ (see [9]). So, $x$ satisfies

$$
\left\{\begin{array}{llrl}
\dot{x}(t) & \in F(t, \mathcal{T}(t) x) & \text { a.e. on }[0, T] ; \\
x(t) & =\varphi(0)+\int_{0}^{t} \dot{x}(s) d s & \forall t \in[0, T] ; \\
x(t) & =\varphi(t) & \forall t \in[-\tau, 0]
\end{array}\right.
$$

The proof is then complete.

\section{Conclusion}

In this paper, an existence result is obtained for first order functional differential inclusions with nonconvex right hand side. The approach used is an adaptation of a reduction method which consists of replacing the problem with delay with a problem without delay and applying the known results in this case.

Acknowledgments. Research supported by the General direction of scientific research and technological development (DGRSDT) under project PRFU No. C00L03 UN180120180001.

\section{REFERENCES}

[1] D. Affane and M. F. Yarou; Second-order perturbed state-dependent sweeping process with subsmooth sets, In: Zeidan D., Padhi S., Burqan A., Ueberholz P. (eds) Computational Mathematics and Applications. Forum for Interdisciplinary Mathematics. Springer, Singapore (2020) 147-169.

[2] D. Affane and M. F. Yarou; General second order functional differential inclusions driven by the sweeping process with subsmooth sets, J. Nonlin. Funct. Anal. Article ID 26 (2020) 1-18. 
[3] D. Affane and M. F. Yarou; Unbounded perturbation to time-dependent subdifferential operators with delay, Electr. J. Math. Anal. Appl. 02(8) (2020) 209-219.

[4] D. Affane and M. F. Yarou; Fixed point approach for differential inclusions governed by subdifferential operators, AIP Conference Proceedings 2183, 060002 (2019); https : // doi.org /10.1063 / 1. 5136157

[5] N. Fetouci and M. F. Yarou; A fixed point approach for a differential inclusion governed by the subdifferential of PLN functions, AIP Conference Proceedings 2183, 060005 (2019); https://doi.org/10.1063/1.5136160

[6] J. P. Aubin, A. Cellina; Differential inclusions, Springer-Verlag, (1984)

[7] M. Bounkhel and M. F. Yarou; Existence results for first and second order nonconvex sweeping process with delay, Portug. Math. 61 (2) (2004) 207-230.

[8] C. Castaing, A. Salvadori and L. Thibault; Functional evolution equations governed by nonconvex sweeping process, J. Nonlin. Conv. Anal. 2(2) (2001) 217-241.

[9] C. Castaing and M. Valadier; Convex Analysis and Measurable Multifunctions, Lecture Note in Math. 580, Springer, Berlin, (1997).

[10] A. Fryszkowski; Continuous selections for a class of non-convex multivalued maps, Studia Math. 76(2) (1983) 163-174.

[11] A. Fryszkowski; Existence of solutions of function-differential inclusion in nonconvex case, Anal. Polonici Math. 45(2) (1985) 121-124.

[12] A. Fryszkowski and L. Gorniewicz; Mixed semicontinuous mappings and their applications to differential inclusions, Set-Valued Anal. 8 (2000) 203-217.

[13] M. F. Yarou; Reduction approach to second order perturbed state-dependent sweeping process, Crea. Math. Infor. 28 (02) (2019) 215-221.

Hanane Chouial,

LmPA laboratory, Department of Mathematics, Jijel University, PB 98, Algeria, Phone: $+213790237071$

Email address: hananechouial@yahoo.com

Mustapha Fateh Yarou,

Lmpa laboratory, Department of Mathematics, Jijel University, PB 98, Algeria, Phone: $+213662419610$

Email address: mfyarou@yahoo.com 